\title{
INTERFRAGMENTARY GAP DISCREPANCY AFTER INTRAORAL ONE-PLATE FIXATION IN MANDIBULAR ANGLE FRACTURE
}

\author{
Hussein Hatem*, Ghada Abdel Monim*, Enas Anter Abd El-Ghaffar ${ }^{* * *}$ and Shaimaa Mohsen Refahee ${ }^{* * * *}$
}

\begin{abstract}
Purpose: Mandibular angle fractures (MAF) recorded many complications following various treatment techniques. Inter-fragmentary gap, strain and movement appear to be responsible for most of the complications with predilection towards the inter-fragmentary gap. This study aimed to calculate and assess the resulted post-treatment inter-fragmentary gap using cone beam computed tomography $(\mathrm{CBCT})$ and its effect on the healing outcome and complications after mini plate fixation at Champy's osteosynthesis line in MAFs.
\end{abstract}

Patients and methods: Twenty-five patients with MAF were subjected to intra-oral open reduction and fixation through semi-rigid min plate fixation near the superior border of the mandible at Champy's osteosynthesis line. The linear inter-fragmentary gap in the mesio-distal plane and the bucco-lingual splay were measured at the inferior border of the mandible after fixation of the fractured bony segments in the final properly aligned position, immediately postoperative, on a CBCT. The healing process was clinically followed for 3 months postoperatively to record any complications.

Results : Uneventful healing was reported in the expected normal duration. At the inferior border of the mandible, the mean mesio-distal inter-fragmentary gap recorded a low value of 0.3 $\mathrm{mm}$. In the bucco-lingual plane, inter-fragmentary lingual splay gap at the inferior border recorded a higher value yet with no clinical effect on healing; $1.8 \mathrm{~mm}$.

Conclusion: Semi-rigid Champy's mini plate is dependable in MAF treatment with predictable acceptable mixed primary and secondary healing outcomes despite the resulted radiographic yet clinically insignificant inter-fragmentary gap and lingual splay at the mandibular inferior border.

KEYWORDS: Mandibular angle fracture, Inter-fragmentary gap, Champy’s miniplate.

* Associate Professor of Oral and Maxillofacial Surgery, Oral and Maxillofacial Surgery Department, Faculty of Dentistry, Cairo University.

** Lecturer of Oral and Maxillofacial Surgery, Oral and Maxillofacial Surgery Department, Faculty of Dentistry, Cairo University.

*** Lecturer of Oral and Maxillofacial Surgery, Oral and Maxillofacial Radiology Department, Faculty of Dentistry, Cairo University.

**** Lecturer of Oral and Maxillofacial Surgery, Oral and Maxillofacial Surgery Department, Faculty of Dentistry, Fayoum University. 


\section{INTRODUCTION}

Mandibular angle fracture (MAF) treatment through accurate reduction and fixation comprise a significant base for facial contour, esthetics and functional occlusion. These treatment goals are fulfilled when the intended reduction successfully brings the fractured bony segments in precise alignment with minimal to no Inter-fragmentary gap between them, along with their stable fixation in this alignment $^{(1)}$.

Many fixation modalities have been suggested and successfully used in MAF varying from the rigid plate fixation and semi-rigid mini plates to the nonrigid inter-maxillary fixation (IMF) wires ${ }^{(2-3)}$. The debate around compression and tension zones of the mandible with the site of plate fixation and its effect on treatment under loading remained throughout the use of these modalities, however, with proved yet variable efficiency of all fixation techniques with no consensus regarding the optimal modality ${ }^{(4-5)}$.

MAF treatment modalities often faces treatment challenges due to its anatomical site, in terms of different functional muscle action along with the absence of teeth distal to the fracture site and absence of dental occlusion in the angle. These attributed strongly to the highest documented complication incidence in treatment of such fractures ${ }^{(6)}$.

Improper reduction and/or inadequate fixation using the recent minimally invasive semi-rigid techniques often lead to an inter-fragmentary gap and soft tissue ingrowth resulting in less bony contact and fragments movement with the inevitable possibility of malunion or even non-union of the fracture ${ }^{(7-8)}$.

Primary bone healing needs direct fragments contact with a minimal gap in between, of less than $0.1 \mathrm{~mm}$, to allow the osteoblasts to easily migrate and deposit bone via "gap healing" through filling it with lamellar bone to form the cortical build. Larger gaps, of 1-2 mm width, induce the formation of fibro-cartilaginous callus which changes into mature cortical bone via over-time remodeling; namely secondary bone healing. While a gap of 1 $\mathrm{mm}$ width comprises a mixed healing procedure between both types ${ }^{(9-10)}$.

Despite the documented fact that interfragmentary movement and strain are principal stimulators in callus formation ${ }^{(11)}$, Ponvel et al ${ }^{(6)}$ and

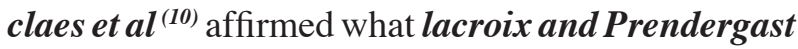
${ }^{(12)}$ and Augat et al ${ }^{(13)}$ previously proved regarding the more important role of inter-fragmentary gap than movement and strain, concluding; the higher gap size and lingual splay, the poorer healing and less mechanical outcome.

To allow cross-sectional viewing and measuring of linear gaps, Cone-beam computed tomography (CBCT), an imperative three-dimensional imaging method which displayed minor skeletal discrepancies and dento-maxillofacial fractures not seen on plain radiographic films, facilitate the multi-planar reformatted cross-sections and threedimensional data ${ }^{(14)}$.

Bearing in mind all this, we aimed to assess the effect of the widely used Champy's mini plate fixation on the inter-fragmentary gap and the consequent impact on fractured segments' contact and healing outcome.

\section{PATIENTS AND METHODS}

This study was performed in faculty of Dentistry, Cairo University in the department of oral and maxillofacial surgery. Ethical approval was obtained from the Cairo University institution and patients' consents were written. The study was recorded in clinical trial (ID: NCT04729036)

This study included twenty-five patients with a mean age of 36 years. They were 17 males and 8 females. All the patients suffered from minimally displaced mandibular angle fracture, diagnosed clinically and radiographically via a panoramic radiograph, and needed intervention through reduction and fixation. (Fig. 1) 
The same surgical team have treated all the patients through open reduction via an intra-oral mucosal flap under general anesthesia with insertion and fixation of a 4-6 holes 2.0 mini-plate and $5 \mathrm{~mm}$ screws at the superior border of the mandible at Champy's osteosynthesis line. (Fig. 2, 3)

\section{Exclusion criteria}

- Patients with multiple mandibular fractures or patients with concomitant maxillary fracture.

- Patients with severely displaced mandibular angle fracture needing extra-oral open reduction and fixation.

- Medically compromised patients and patients with abnormalities that impair bone healing.

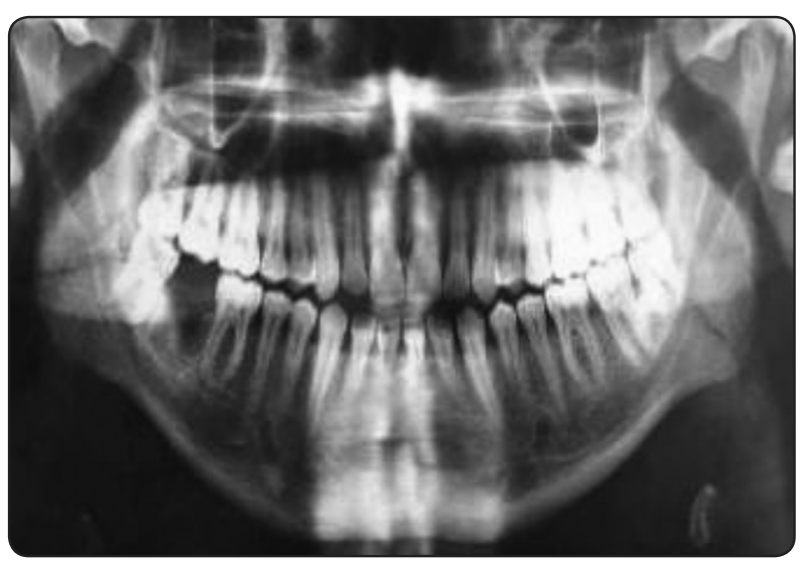

Fig. (1): A panoramic radiograph showing the preoperative fractured mandibular angle

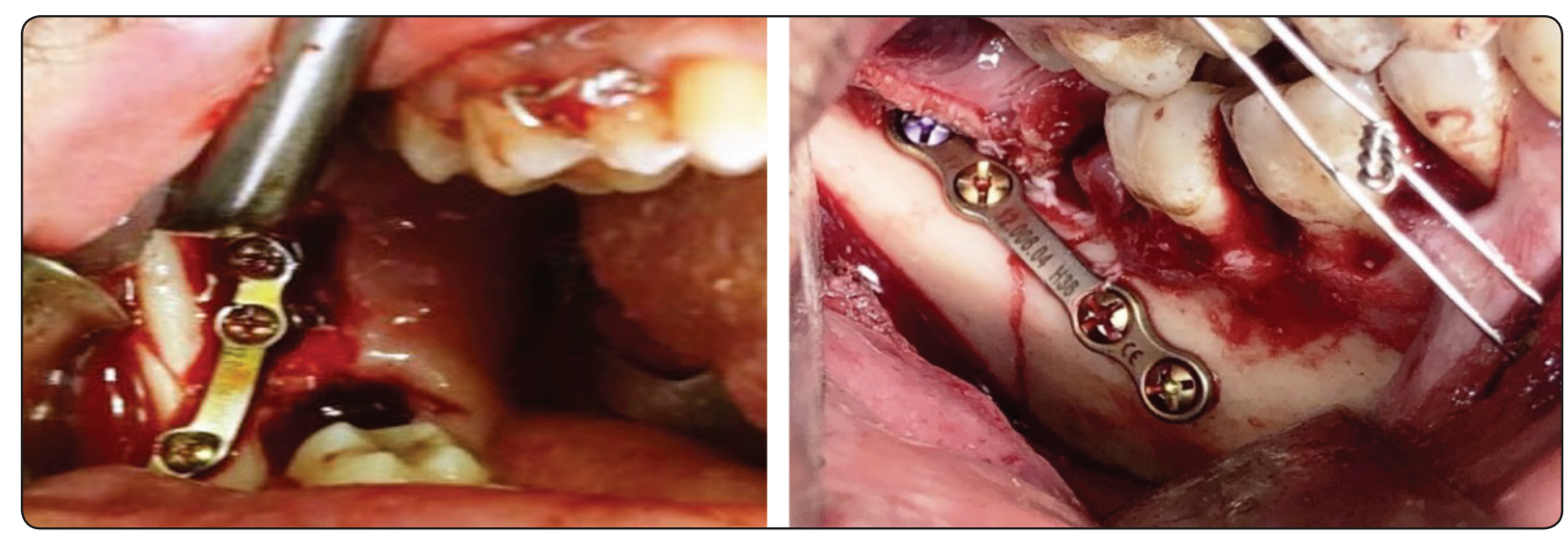

Fig. (3): Photographs showing the intra-operative fixation of Champy's mini plate.

\section{Radiographic analysis}

An immediate post-surgical CBCT was done by Planmeca promax 3D Mid ${ }^{\mathrm{R}}$ machine to measure the mesio-distal plane discrepancy in terms of linear width of inter-fragmentary gap and the linear depth of bucco-lingual gap in terms of lingual splay at the inferior border between the fractured mesial and proximal segments.

Using Planmeca Romexis viewer ${ }^{\mathrm{R}}$ 5.2.0 software, the same operator generated the required readings twice for each patient at different times to avoid any outer influences and the final average value for each reading was recorded. The mean values were then calculated to assess the inter-fragmentary gap between the fractured segments after fixation which indicates the success of the fixation method and

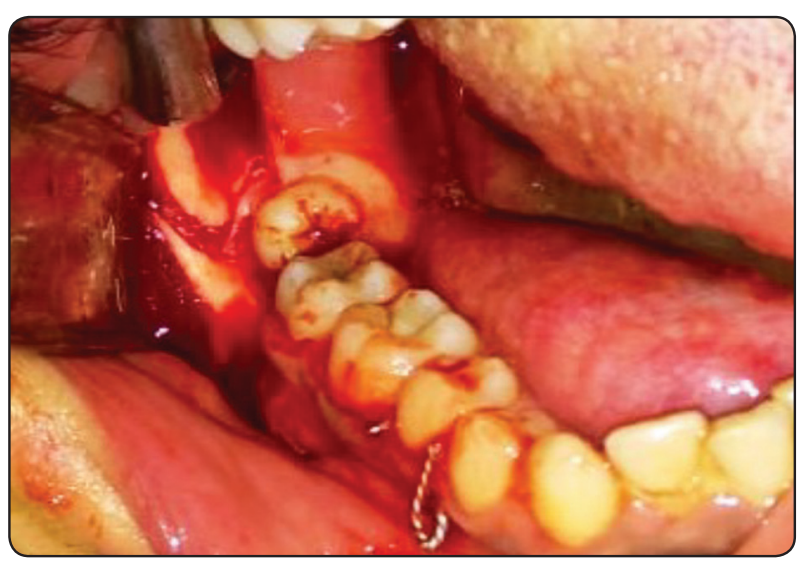

Fig. (2): A photograph showing the intra-operative fractured mandibular angle 
the type of bone formed through different healing patterns namely, primary, mixed or secondary healing with a gap of $<0.1 \mathrm{~mm}$, up to $1 \mathrm{~mm}$ and 1-2 mm or more respectively, according to the gap record scale documented in the literature ${ }^{(9,15)}$.

\section{Clinical assessment}

All patients were followed up for a period of 3 months to assess the clinical healing and record any complication or patient complain.

\section{RESULTS}

Twenty-five patients were included in this study. They were all suffering from MAF which needed intervention in the form of reduction and fixation. The included patients were 17 males and 8 females aging from 23-45 with a mean age of 36 years old.
All patients underwent intra-oral open reduction and semi-rigid mini plate fixation using Champy's superior border plating.

Along the treatment duration, uneventful healing in the expected normal duration with no complications or complaints reported except for the normal post-surgical edema and pain. No reported dehiscence or delayed/non-healing were documented.

The mean mesio-distal inter-fragmentary gap at the inferior border showed low mean records of 0.3 millimeters ( $\mathrm{mm}$ ) width. In the bucco-lingual plane, a lingual inter-fragmentary splay gap at the inferior border recorded a higher mean value of $1.8 \mathrm{~mm}$ yet with a clinical insignificant effect on healing. (Fig. 4)
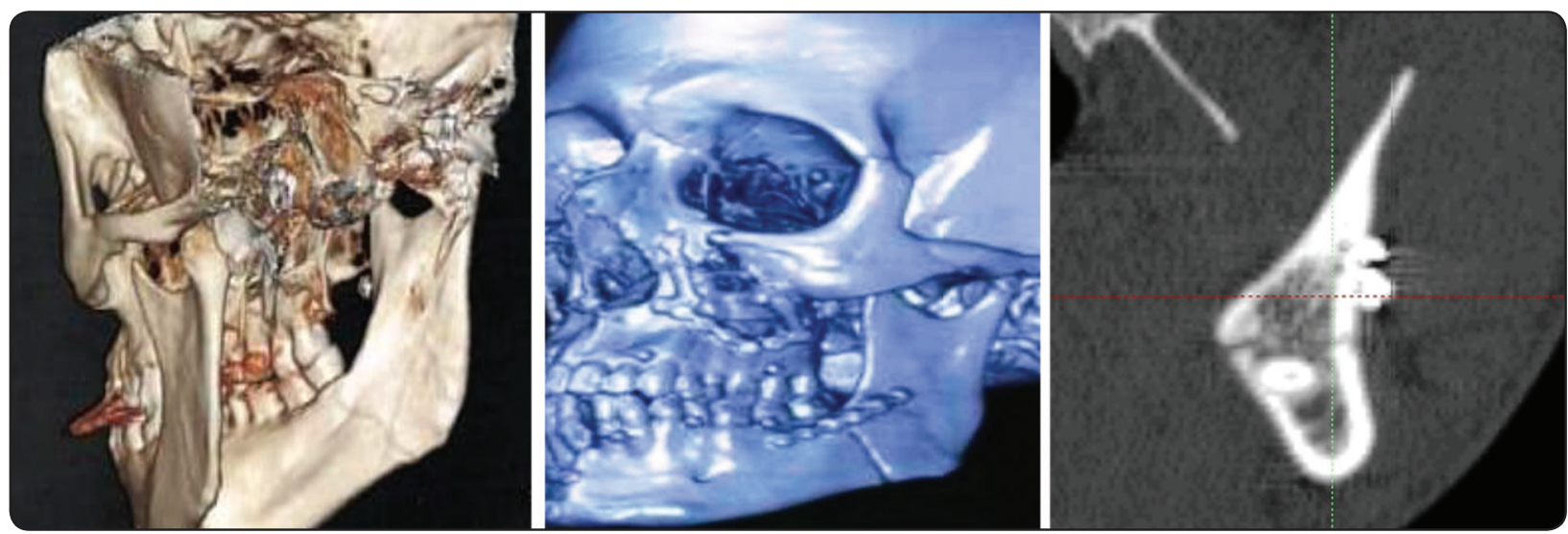

Fig. (4): CBCT images showing the cross-sectional inter-fragmentary gap after fracture fixation.

\section{DISCUSSION}

Many treatment options were established in the literature regarding the reduction and fixation of MAFs. These ranged from the non-rigid IMF using wires, Champy's minimally invasive semirigid mini plate to the invasive needing approach dynamic plates ${ }^{(2-3)}$.
Despite of the proved efficacy of all these techniques, different drawbacks appeared through the treatment process of each modality depending on the faced challenges, as the absence of teeth in the distal bony segment beyond the fracture line, the several multi-directional muscles' actions on the fractured segments especially the distal segment and the masticatory function ${ }^{(2-3,16)}$. 
Nevertheless, the widely used Champy's superior border mini plate have been highly recommended for its intra-oral minimally invasive approach and ease of application with the beneficial avoidance of closing the mouth and other known disadvantages of IMF along with the prevention of the extraoral invasive flaps in utilizing the inferior border rigid plates. However, regarding Champy's method, a debate has been aroused concerning the mandibular fractured segments' direct contact and the produced inter-fragmentary gap ${ }^{(17-19)}$.

In opposition to Perren ${ }^{(11)}$ and Ribeiro et al ${ }^{(20)}$ who stated that the strain determines the tissue type formed in the gap, this study addressed the interfragmentary gap and lingual splay for their higher importance in healing and bone formation type over the accompanied inter-fragment movement and strain which was agreed upon with other authors ${ }^{(10,15)}$.

Logically, as inter-fragmentary gap widens, the more healing time is needed for adequate stability and the more inter-fragment movement with less bending stiffness. Side effects of what is considered a large gap included the un-aesthetic widening of the face, ingrowth of soft tissue between the fractured segments, infection, fractured segments' movement and delayed or even non-union and absence of healing ${ }^{(15-21)}$.

Due to all this, this study approached this widely and nearly routinely used treatment modality to assess the resultant inter-fragmentary gap and delineate its effect on the healing outcome of MAF.

According to the literature, CBCT has been reported to be superior to plain radiography with an approaching accuracy to CT. It was reported as the modality of choice for mandibular trauma imaging for its three dimensional hard tissue data in different planes which can be reformatted and threedimensionally reconstructed, hence the utilized CBCT in this study in measuring the different planes' inter-fragmentary gap ${ }^{(22-23)}$.

In consistence with several surgeons, no dehiscence or any patient complains, or complications were reported other than the expected post-surgical edema and temporary pain ${ }^{(15-20)}$.

\section{Similar to Wang et al ${ }^{(15)}$ and Prasad et al (24),} the recorded mesio-distal gap between the segments was less than $1 \mathrm{~mm}$ in all patients, with a mean of $0.3 \mathrm{~mm}$, yet with a higher lingual splay in the bucco-lingual plane between with a mean value of $1.8 \mathrm{~mm}$. According to their followed scale, the resulted values, despite of the single plane superior fixation, produced an undisturbed mesio-distal mixed primary and secondary healing with a buccolingual secondary healing patterns allowing a mixed form of healing which explains the normal resulted expected healing and duration.

According to our results, the mesio-distal gap was less than the bucco-lingual splay. This may be due to the acting muscles, especially the medial pterygoid muscle, on the distal fractured bony segment which pulls it upward and medial to allow for the medial displacement and higher splay recorded value, while the mesio-distal low gap value was attributed to the approaching fractured segments at the inferior border of the mandible under loading stated by kroon et al ${ }^{(18)}$ and Mannan et $\boldsymbol{a l}^{\left({ }^{(19)}\right.}$ namely, the compression zone, preventing movement or widening of the present gap allowing for an undisturbed healing.

Thus, Champy's single superior mini plate treatment modality under study has resulted in the expected acceptable clinical healing outcome and minimal complains and complications proving its efficiency in MAF treatment in accordance to the documented literature, in spite of the recorded considerably high inter-fragmentary gap particularly in the form of lingual splay producing a mixed primary and secondary healing pattern.

\section{CONCLUSION}

Semi-rigid Champy's mini plate is a dependable line of treatment in MAF with satisfying healing outcomes despite the recorded insignificant interfragmentary gap splay. 


\section{RECOMMENDATIONS}

In order to further understand the interfragmentary gap effect on healing, an extended invitro study is recommended to address the created histological type of bone throughout the healing duration.

\section{Declaration of conflicting interests}

The authors declare that there is no conflict of interest.

\section{Funding resources}

There were no received funding sources.

\section{REFERENCES}

1. Moore E, Bayrak S, Moody M, Key JM and Vural E. Hardware removal rates for mandibular angle fractures: comparing the 8-hole strut and Champy plates. J Craniofac Surg; 24: 163-5, 2013.

2. Chrcanovic BR. Fixation of mandibular angle fractures: clinical studies. Oral Maxillofac Surg; 12: 45, 2012.

3. Chrcanovic BR. Fixation of mandibular angle fractures: in vitro biomechanical assessments and computer-based studies. Oral Maxillofac Surg; 17:251-68, 2013.

4. Sadhwani B and Anchlia S. Conventional $2.0 \mathrm{~mm}$ miniplates versus 3-D plates in mandibular fractures. Annals of Maxillofacial Surgery; 3: 2, 2013.

5. Singh RK, Pal US, Agrawal A, and Singh G. Single miniplate osteosynthesis in angle fracture. Natl J Maxillofac Surg; 2:47-50, 2011

6. Ponvel K, Panneersevam E, Balasubramanian S, and Raja $\mathrm{KK}$ : Evaluation of labial versus labio-inferior lines of osteosynthesis using 3D miniplate for fractures of anterior mandible: A finite element analysis with a pilot clinical trial. Chinese Journal of Traumatology; 22: 261-269, 2019.

7. Lee U, Rojhani A, Herford A, and Thakker J. Immediate versus delayed treatment of mandubilar angle fractures: A stratified analysis of complications. J Oral Maxiloofac Surg; 73: 1-11, 2016.

8. Seemann R, Schicho K, Wutzl, et al: Complication rates in the operative treatment of mandibular angle fractures: A 10-year retrospective. J Oral Maxillofac Surg; 68: 64750,2010 .

9. Kwong Fn, Mitchel B, and harris MB: Recent developments in the biology of fracture repair. J Am Acad orthop Surg; 63: 619-25, 2008.

10. Claes L, Eckert-Hubner K, and Augat p: The fracture gap size influences the local vascularization and tissue differentiation in callus healing. J Langenbecks Arch Surg; 388: 316-22, 2003.
11. Perren SM. The fracture healing. The evolution of our understanding. Acta Chir Orthop Traumatol; 7: 241-8, 2008.

12. Lacroix D and Prendergast PJ: A mechano-regulation model for tissue differentiation during fracture healing: analysis of gap size and loading. J Biomech; 35: 1163-71, 2002

13. Augat P, Margevicius K, Simon J, Wolf S, Suger G, and Claes L: Local Tissue Properties in Bone Healing: Influence of Size and Stability of the Osteotomy Gap. J Orthopedic Research; 16: 175-481, 1998.

14. Choudhary AB, Motwani MB, Degwekar SS, Bhowate RR, Banode PJ, Yadav AO, et al. Utility of digital volume tomography in maxillofacial trauma. J Oral Maxillofac Surg; 69(6):135-40, 2011.

15. Wang R, Liu Y, Wang JH, and Baur DA: Effect of interfragmentary gap on mechanical behavior of mandibular angle fracture with three fixation designs-a finite element analysis. J Plastic, Reconstr \& aesthetic Surg; 10: 26, 2016.

16. Gear AJ, Apasova E, Schmitz JP. Treatment modalities for mandibular angle fractures. J oral Maxillofac Surg; 63: 655-63, 2005.

17. Champy M and Pape HD. The strasbourg miniplate osteosynthesis. In: Kruger E, Schilli W, editors. Oral and Maxillofacial Traumotology. vol. 2. Berlin: Quintessence; 19: 43, 1986.

18. Kroon F, Mathisson M, Cordey J, and Rahn B. The use of miniplates in mandibular fractures. J Cranio Maxillofac Surg; 19:199-204, 1991.

19. Mannan R, Farooq A, and Mukhtar H. Comparison of Single vs Double Miniplates in the Management of Mandibular Angle Fractures. P J M H S; 12: 662, 2018.

20. Ribeiro M, Lauria A, Sato F, Moreira W. Biomechanical analysis on different fixation techniques for treatment of mandibular body fractures. Braz J Oral Sci; 12: 80-3, 2013.

21. Rahpeyma A, Khajehahmadi S, Abdollahpour S: Mandibular symphyseal/parasymphyseal fracture with incisor tooth loss: preventing lower arch constriction. Craniomaxillofac Trauma Reconstr; 9: 15-19, 2016

22. Kaeppler G, Cornelius CP, Ehrenfeld M, Mast G. Diagnostic efficacy of cone-beam computed tomography for mandibular fractures. Oral Surg Oral Med Oral Pathol Oral Radiol. 2013;116(1):98-104.

23. Parrone MT, Bechara B, Deahl ST 2nd, Ruparel NB, Katkar R, Noujeim M. Cone beam computed tomography image optimization to detect root fractures in endodontically treated teeth: an in vitro (phantom) study. Oral Surg Oral Med Oral Pathol Oral Radiol. 2017;123(5):613-20.

24. Prasad R, Thangavelu K, John R. The role of 3D plating system in mandibular fractures: a prospective study. $\mathrm{J}$ Pharm Bioallied Sci; 5: S10-13, 2013. 